\title{
Contribution of caspase-independent pathway to apoptosis in malignant glioma induced by carbon ion beams
}

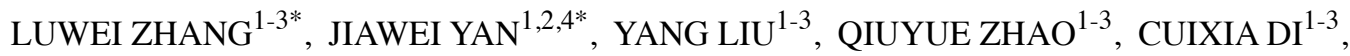 \\ SUN CHAO ${ }^{1-3}$, LI JIE ${ }^{5}$, YUANYUAN LIU ${ }^{1-3}$ and HONG ZHANG ${ }^{1-3}$

\begin{abstract}
${ }^{1}$ Department of Heavy Ion Radiation Medicine, Institute of Modern Physics, Chinese Academy of Sciences;
${ }^{2}$ Key Laboratory of Heavy Ion Radiation Biology and Medicine of Chinese Academy of Sciences; ${ }^{3}$ Key Laboratory of Heavy Ion Radiation Medicine of Gansu Province, Lanzhou, Gansu 730000; ${ }^{4}$ University of Chinese Academy of Sciences, Beijing 100039; ${ }^{5}$ School of Stomatology, Lanzhou University, Lanzhou, Gansu 730000, P.R. China
\end{abstract}

Received August 9, 2016; Accepted December 28, 2016

DOI: $10.3892 /$ or.2017.5529

\begin{abstract}
High linear energy transfer (LET) carbon ion beam (CIB) is becoming the best tool for external radiotherapy of inoperable tumors because of its greater cell killing than conventional low LET gamma or X-rays. In the present study, whether the caspase-independent pathway exerts the important contribution in CIB-induced cell apoptosis was explored. Herein we showed, despite the absence of caspase activity using a pan caspase inhibitor Z-VAD-FMK, that apoptosis induced by high LET CIB were clearly observed in the glioma cells. Simultaneously, the increased 8-OHdG level, PARP-1 activity and AIF translocation occurred in response to CIB irradiation. Moreover, it was distinctly higher in the nuclear translocation frequency along with PARP-1 activation when the caspase protease cascade was suppressed in the irradiated glioma cells. Nuclear colocalization between PARP-1 and AIF as well as a positive association of the PARP-1 mRNA expression with AIF translocation frequency indicated that PARP-1 activation controlled the translocation of AIF to the nucleus. Our findings strongly demonstrated that caspase-independent cell apoptosis provided a prominent compensation in the glioma cell death involving the PARP-1/AIF signaling pathway at $24 \mathrm{~h}$ after CIB
\end{abstract}

Correspondence to: Professor Hong Zhang, Department of Heavy Ion Radiation Medicine, Institute of Modern Physics, Chinese Academy of Sciences, 509 Nanchang Road, Lanzhou, Gansu 730000, P.R. China

E-mail: zhangh@impcas.ac.cn

${ }^{*}$ Contributed equally

Abbreviations: AIF, apoptosis inducing factor; PARP-1, poly(ADPribose) polymerase-1; CIB, carbon ion beam; LET, linear energy transfer; DAPI, 4', 6-diamidino-2-phenylindole; FITC, fluorescein isothiocyanate; PI, propidium iodide

Key words: apoptosis, CIB, caspase-independent pathway, apoptosis inducing factor, PARP-1, glioma exposure, and likely triggered by oxidative damage to DNA. The knowledge on the molecular mechanism of AIF-mediated cell death may be very useful for the improvement of the therapeutic efficacy of malignant gliomas with heavy charged particles.

\section{Introduction}

Radiation therapy has remained the mainstay of treatment for glioblastoma, but these tumors are often associated with radioresistance (1). Heavy ions represent the best tool for various tumors, especially for radioresistant tumors mediated by hypoxia, due to several advantages over conventional radiotherapy (gamma and X-rays) such as an inverted depthdose distribution, a higher relative biological effectiveness, a reduction in oxygen enhancement ratio, less variation in cell cycle related sensitivity and cells having decreased ability to repair (2).

Cell apoptosis is a key mechanism through which ionizing radiation kills tumor cells via two principal signaling pathways, the extrinsic death pathway involving the ligation of death receptors and the intrinsic death pathway initiated at the mitochondrion (3). Among them, intrinsic apoptosis seems to play a key part in the regulation of susceptibility of tumor cells to radiation (4). Caspases, a family of cysteine proteases, have been recognized as important mediators of apoptosis in the intrinsic apoptotic cascade. There is accumulating evidence indicating that heavy ion radiation can effectively trigger apoptosis as described in previous findings $(5,6)$ which can be through activation of caspase-3 and caspase-9 (7); however, so far little information is available on the caspase-independent form of apoptosis.

Apoptosis-inducing factor (AIF), a mitochondrion-localized flavoprotein initially discovered as a caspase-independent death effector, is released in response to death stimuli, and subsequently results in chromatin condensation and largescale fragmentation of DNA (8-10). There is definite evidence demonstrating that AIF has been shown to associate well with induction of cell death after ischemia/reperfusion (11), hypoglycemia (12), brain trauma (13) and neurodegenerative diseases (14). 
Poly(ADP-ribose) polymerase 1 (PARP-1), the major isoform of the poly(ADP-ribose) polymerase family, is a constitutive nuclear and mitochondrial protein with wellrecognized roles in base excision repair and DNA strand break repair (15). Over activation of PARP-1 is required for the translocation of AIF from the mitochondria to nucleus as an important activator of caspase-independent cell death (16-18). PARP-1 activity was reported to affect the radiation sensitivity of tumor cells by manipulation of the DNA repair processes (19), cell cycle (20) and autophagy (21) following exposure to ionizing radiation.

Among all high-LET heavy ion radiations, CIB is becoming popular and excellent in treatment of malignant tumors. In the current study we present preliminary data which could help to clarify the role of caspase-independent manner in CIB-induced glioma cell death by means of caspase inhibitor Z-VAD-FMK. Furthermore, PARP-1/AIF pathway might contribute to the development of more effective cancer radiotherapy. This study provides the supplement to the apoptosis mechanisms caused by the heavy ion radiation and demonstrates a potential benefit of therapeutic strategies for high-LET particle therapy.

\section{Materials and methods}

Cell culture and irradiation. Human glioblastoma multiform (GBM) U251 cell line was obtained from China Center for Type Culture Collection (CCTCC), Wuhan, China. Cells were maintained in DMEM medium supplemented with $10 \%$ FBS, $100 \mathrm{U} / \mathrm{ml}$ penicillin and $100 \mathrm{U} / \mathrm{ml}$ streptomycin in a humid atmosphere of $5 \% \mathrm{CO}_{2}$ and $95 \%$ air at $37^{\circ} \mathrm{C}$. U251 cells were irradiated with $2 \mathrm{~Gy}{ }^{12} \mathrm{C}^{+}$ions at room temperature. A carbon ion beam of $350 \mathrm{MeV} / \mathrm{u}$ was supplied by the Heavy Ion Research Facility in Lanzhou (HIRFL) at the Institute of Modern Physics, Chinese Academy of Sciences (Lanzhou, China). The dose rate was adjusted to be approximately $0.2 \mathrm{~Gy} / \mathrm{min}$.

Apoptosis assay. Hoechst 33258 staining was performed with a few modifications. Briefly, after exposure to radiation, confluent cells were incubated for $24 \mathrm{~h}$ at $37^{\circ} \mathrm{C}$ in 6 -well plates. Then these cells were fixed with $4 \%$ paraformaldehyde for $30 \mathrm{~min}$ at RT and washed once with PBS. Fixed cells were stained with Hoechst 33258 of $50 \mathrm{ng} / \mathrm{ml}$ and incubated for $30 \mathrm{~min}$ at RT and washed with PBS. Apoptotic cells were identified by condensation and fragmentation of nuclei examined by fluorescence microscopy.

Quantification of apoptotic cells was obtained using the Annexin V-FITC detection kit (Invitrogen, Eugen, Oregon, USA) according to the manufacturer's protocol. Following controls were used to set up compensation and quadrants: unstained cells and cells stained with FITC-Annexin V or with PI alone. The apoptotic/necrotic cell population was analyzed with a FACS Calibur flow cytometer (Becton Dickinson, Franklin Lakes, NJ, USA).

Flowsight data acquisition and analysis. Acquisition speed was set up to low speed and the highest resolution, an automated condition provided in Flowsight (Amnis/Merck Millipore, Darmstadt, Germany). Approximately 1000-5000 cells were acquired. Channel 5 was used to acquire DRAQ5 and channel 2 was used to detect Alexa Fluor 488. Data were analyzed in IDEAS software after compensation of single color control samples using a compensation matrix. The frequency of AIF translocation to the nuclei was analyzed using the nuclear translocation application wizard in IDEAS software.

Gene expression analysis. Total RNA was extracted from glioma cells at $24 \mathrm{~h}$ after CIB irradiation using the TRIzol Reagent (Invitrogen Life Technologies, Carlsbad, CA, USA) according to the manufacturer's instructions. The cDNA was synthesized from $1 \mathrm{mg}$ total RNA using RT reagent kit with gDNA Eraser (Takara, Tokyo, Japan) following the manufacturer's protocols. Real-time polymerase chain reaction (quantitative PCR) was carried out the SYBR Premix EX Taq II kit (Takara, Dalian, China) on an FTC-3000+ instrument (Funglyn Biotech Inc., Toronto, ON, Canada). Gene expression was detected using qPCR primers for PARP-1 and $\beta$-actin which was used to normalize the mRNA and cDNA quantity and quality. Sequences of the primers are as follows: PARP-1 sense, 5'-TAGGCATGATTGACCGCTGG-3', and antisense, 5'-ACCATGCCATCAGCTACTCG-3'; $\beta$-actin sense, 5'-TGA GCGCAAGTACTCTGTGTGGAT-3', and antisense, 5'-TAGA AGCATTTGCGGTGCACGATG-3'. The PCR program was denatured at $95^{\circ} \mathrm{C}$ for $30 \mathrm{sec}$, followed by 40 cycles of $95^{\circ} \mathrm{C}$ for $5 \mathrm{sec}, 60^{\circ} \mathrm{C}$ for $30 \mathrm{sec}$. The fold changes of gene expression of the treatment groups were calculated by the $2^{-\Delta \Delta \mathrm{Ct}}$ method.

Immunofluorescence. GBM U251 cells were fixed in $4 \%$ paraformaldehyde for $10 \mathrm{~min}$ and permeabilized with $0.2 \%$ Triton X-100 for $10 \mathrm{~min}$. Cells were blocked for $1 \mathrm{~h}$ with TBST containing $0.1 \%$ BSA followed by a $1 \mathrm{~h}$ incubation with rabbit polyclonal or mouse polyclonal antibody, PARP-1 (1:400, Santa Cruz Biotechnology, Inc., Santa Cruz, CA, USA) and AIF (1:400, CapitalBio, Beijing, China). The primary antibody was detected with Alexa Fluor-555 donkey anti-rabbi secondary antibody (1:1000, Invitrogen, Carlsbad, CA, USA) or Alexa Fluor-488 goat anti-mouse (1:1000, Invitrogen). The cells were counterstained with DAPI (Vector Laboratories, Burlingame, CA, USA). Confocal images were acquired using a Zeiss LSM-700 confocal microscope. Fluorescence intensity was quantificationally analyzed using ZEN 2010 software (Carl Zeiss).

Statistical analysis. Statistical analysis for each of the studied parameters was performed on the means of the data obtained from at least 3 independent experiments. Data are presented as means \pm SD. Student's t-test program in Microsoft Excel was used to detect statistical significance. A P-value $<0.05$ was selected as a criterion for a statistically significant difference.

\section{Results}

Cell apoptosis. Apoptotic cells were determined by Hoechst 33258 and Annexin V/PI staining $24 \mathrm{~h}$ after CIB irradiation. As illustrated in Fig. 1, it shows radiation-induced augmentation in the proportion of apoptotic cells which displayed the typical morphological features of cell death such as cell shrinkage, nuclear condensation and formation of pyknotic bodies of condensed chromatin. Z-VAD-FMK 

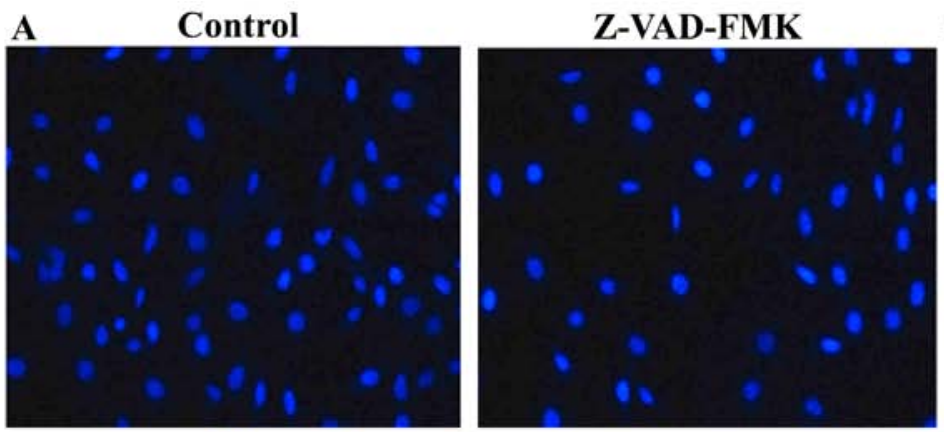

B

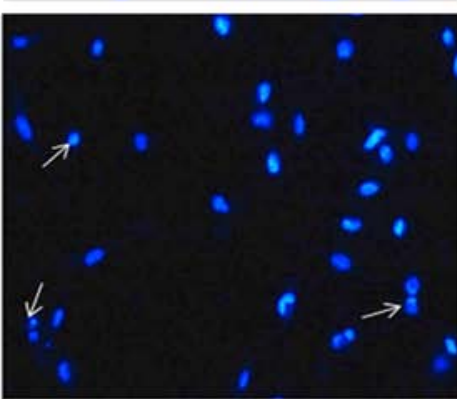

CIB

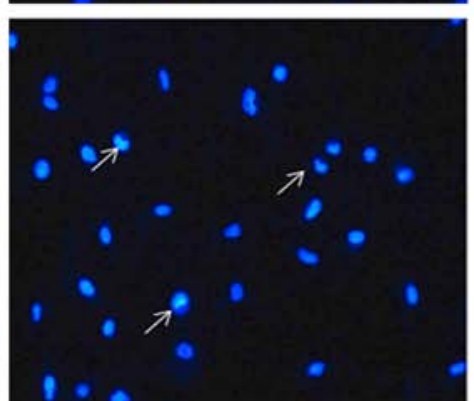

Z-VAD-FMK+CIB

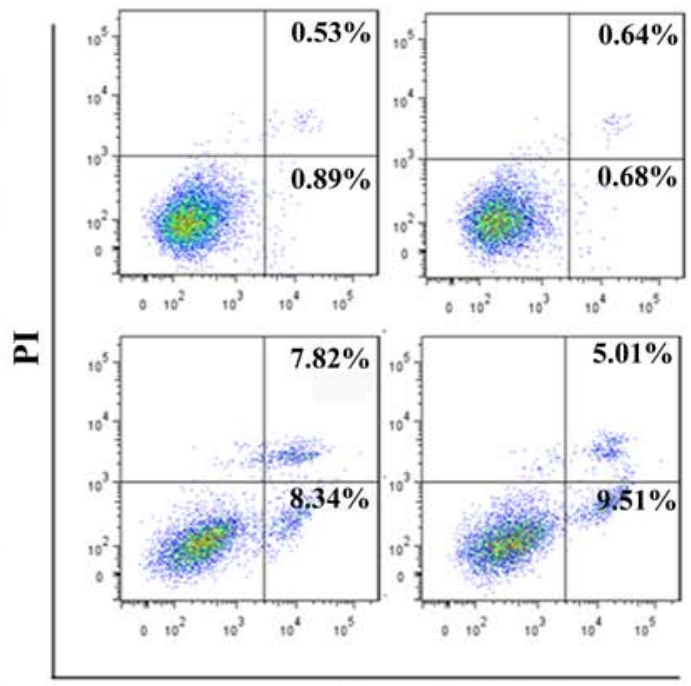

AnnexinV

Figure 1. Effect of Z-VAD-FMK and CIB on the apoptosis of U251 cells. Cells were incubated with $50 \mu \mathrm{M}$ Z-VAD-FMK before 2 Gy CIB irradiation. (A) Annexin V-FITC/PI staining and flow cytometric determination of apoptosis in cells. Percentage denotes proportion of apoptotic cells in right lower quadrant and right upper quadrant. (B) The nuclear morphological changes by Hoechst staining were assessed under a fluorescence microscope (original magnification, $\mathrm{x} 200)$.

A

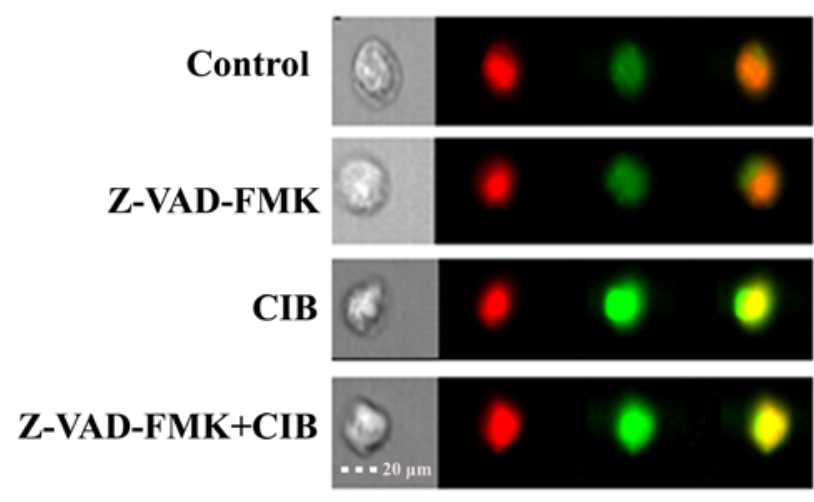

B

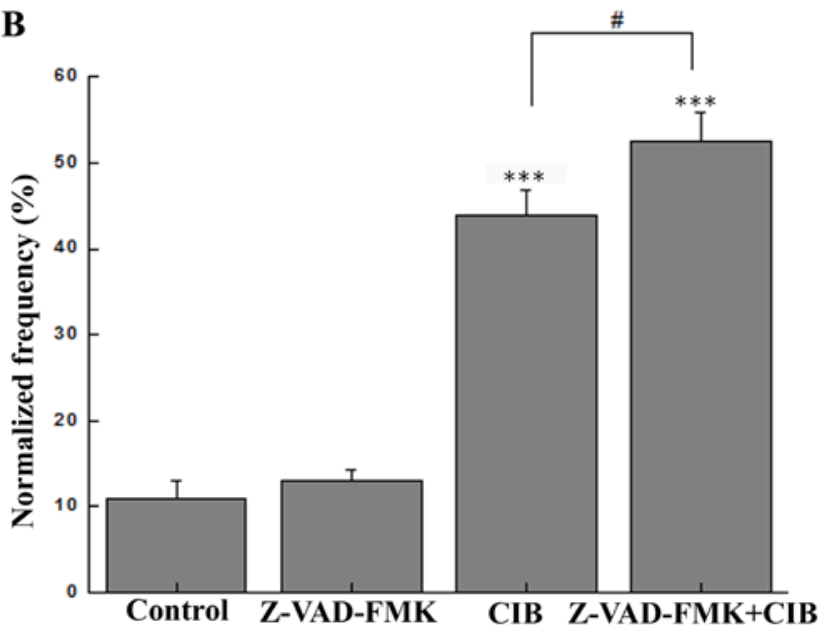

Figure 2. AIF nuclear translocation of U251 cells with/without Z-VAD-FMK treatment (50 $\mu \mathrm{M}$ ) before 2 Gy CIB irradiation using a FlowSight. (A) Representative images of AIF translocation into the nucleus. Green color denotes AIF protein and red color indicates nucleus. The merged color regions (orange) represent the AIF translocation into nucleus. (B) The nuclear translocation frequency of AIF protein is presented as means \pm SD from three independent experiments. ${ }^{* * * *} \mathrm{P}<0.001$ compared to the control and ${ }^{\#} \mathrm{P}<0.05$ compared to 2 Gy irradiation alone.

$(50 \mu \mathrm{M})$, pan inhibitor of caspases, blocked caspase-mediated mitochondrial membrane depolarization. Interestingly, no distinct difference was detected in the cellular apoptosis of cells pretreated with Z-VAD-FMK followed by CIB irradiation (14.52\%) and cells treated with CIB alone (16.16\%), indicating that CIB can trigger apoptosis in U251 cells via an alternative caspase-independent pathway.

AIF translocation. AIF, an important caspase-independent death effector, translocates from mitochondria to the cytosol as well as the nucleus when apoptosis is induced. To explore the detailed mechanism of cell death program elicited by CIB, AIF translocation was investigated by Imaging Flow Cytometry, which is closely linked to the caspase-independent apoptosis. Green color denotes AIF protein and red color denotes nucleus. The merged color regions with high density represent the AIF translocation into nucleus. Herein, CIB radiation promoted the AIF nuclear translocation in glioma cells as observed in Fig. 2A. Amnis data exhibit that the nuclear translocation frequency of AIF protein was increased 3.96- or 4.81-flod in the CIB alone group or in the combination with Z-VAD-FMK group compared with the control 

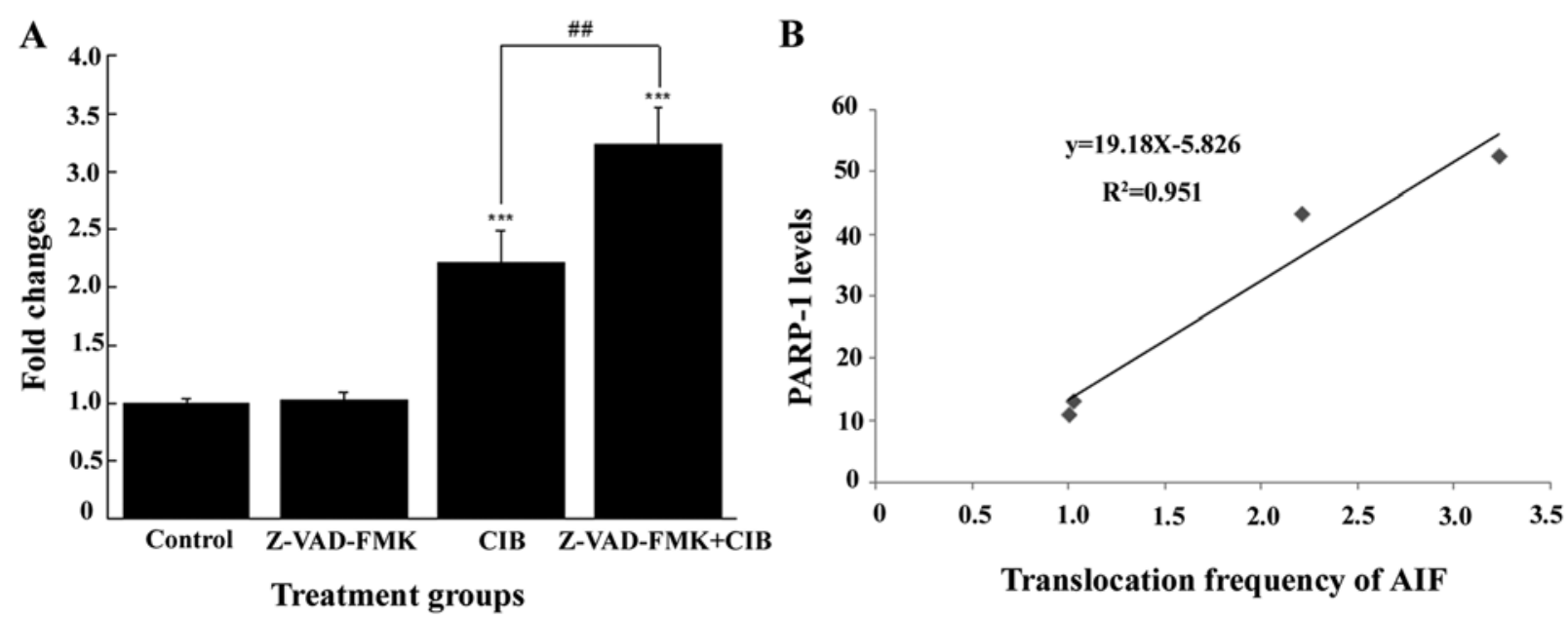

Figure 3.Z-VAD-FMK enhances PARP-1 transcript expression post $24 \mathrm{~h}$ irradiation. (A) The relative levels of PARP-1 in the CIB irradiated cells as measured by real-time PCR. (B) The relationship between PARP-1 mRNA levels and translocation frequencies of AIF after Z-VAD-FMK treatment combined with 2 Gy CIB irradiation. Data are presented as means \pm SD from three independent experiments. ${ }^{* * * *} \mathrm{P}<0.001$ compared to the control and ${ }^{\# \#} \mathrm{P}<0.01$ compared to 2 Gy irradiation alone.

\section{Control}
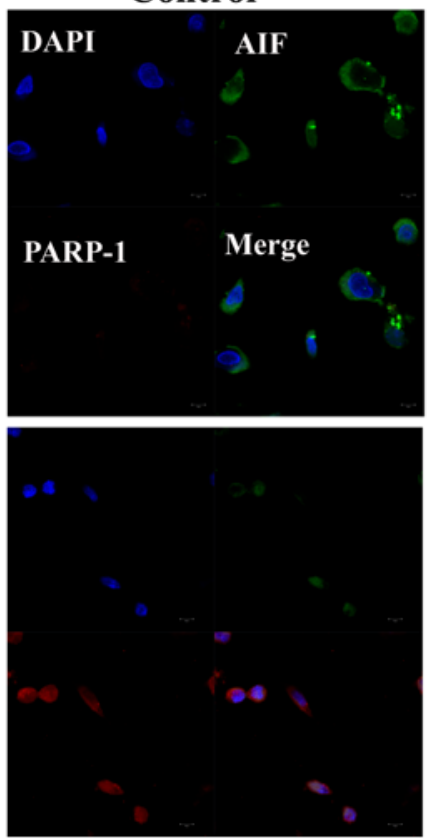

CIB
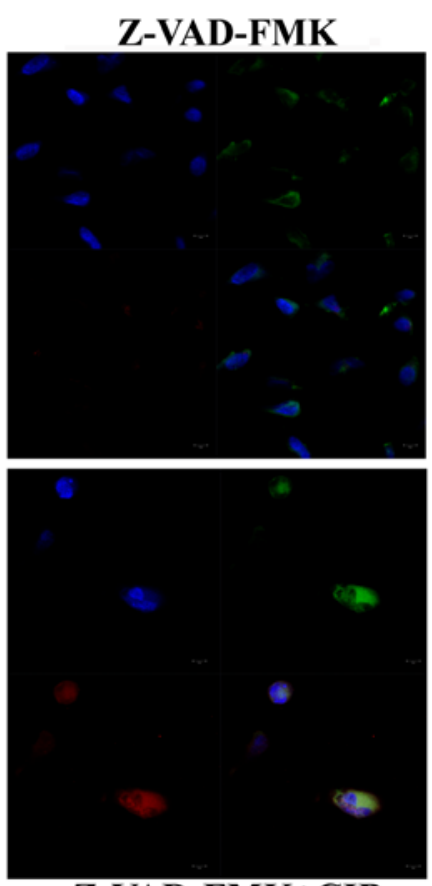

Z-VAD-FMK+CIB

Figure 4. PARP-1 and AIF in U251 cells with/without Z-VAD-FMK treatment $(50 \mu \mathrm{M})$ before CIB irradiation. Cells were administered with $50 \mu \mathrm{M}$ Z-VAD-FMK prior to 2 Gy CIB irradiation Confocal microscopy was used to visualize the intracellular colocalization of AIF (green fluorescence) and PARP-1 (red fluorescence).

group, respectively. Furthermore, incubation with or without Z-VAD-FMK had a significant difference on the AIF nuclear translocation $(\mathrm{P}<0.05$, Fig. $2 \mathrm{~B})$. These data imply that the inhibition activation of caspase may lead to AIF translocation with compensatory enlargement.

PARP-1 mRNA expression. To ascertain the cause of the AIF translocation in the irradiated glioma cells, we determined the PARP-1 status which is the decisive molecule responsible for the translocation of AIF from the mitochondria to nucleus. As

given in Fig. 3, the data from qRT-PCR exhibited that PARP-1 expression level was significantly elevated by irradiation with CIB compared with the control $(\mathrm{P}<0.001)$ at $24 \mathrm{~h}$ postirradiation. Remarkably, CIB-induced PARP-1 activation is higher 1.46-fold in the presence of Z-VAD-FMK than in the absence of Z-VAD-FMK, suggesting that caspase inhibition could improve the PARP-1 activation. In addition, a positive correlation between AIF translocation with PARP-1 mRNA level was found $\left(\mathrm{y}=19.18 \mathrm{x}-5.826, \mathrm{R}^{2}=0.951\right)$ in Z-VAD-FMKintervened glioma cells.

Interactions of PARP-1 with AIF. To further characterize the role of PARP-1 activity in AIF translocation of glioma cells following CIB treatment, we confirmed the interactions of AIF and PARP-1 by immunofluorescence analysis. As shown in the Fig. 4, it is evident that the colocalization of PARP-1 and AIF in the nucleus was distinctly potentiated in the cells irradiated with CIB as well as in those supplemented with Z-VAD-FMK prior to CIB irradiation. In addition, immunofluorescence result showed that CIB markedly increased the PARP-1activation, which was possibly in response to the accumulated DNA damage.

Oxidative DNA damage. The percentage of U251 cells possessing oxidative damage to their DNA was assessed using 8-OHdG as the marker. As seen in Fig. 5, CIB radiation enhanced the oxidation of DNA compared to the untreated control. The signal intensity of 8-OHdG-positive cells prominently increased $24 \mathrm{~h}$ after irradiation $(\mathrm{P}<0.01)$, with most immunoreactivity in the perinuclear region of the cytoplasm (Fig. 5A). The 8-OHdG content was higher in the mitochondrial than the nuclear fraction. Moreover, the supplement of Z-VAD-FMK had no significant effect on glioma cells in terms of the percentage 8-OHdG.

\section{Discussion}

Currently, a few studies indicate that inhibition of caspase did not affect apoptosis in malignant glioma cells and various 
A

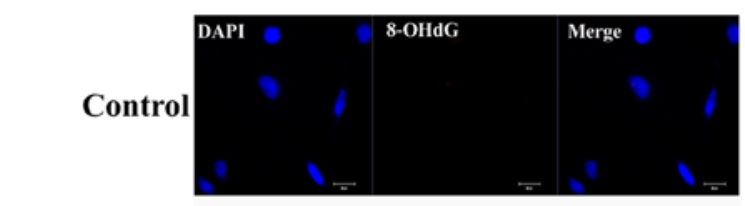

Z-VAD-FMK+CIB

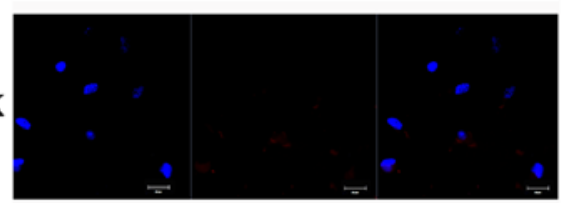

Z-VAD-FMK

CIB
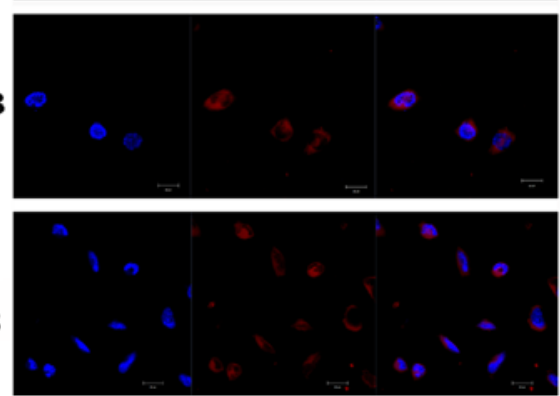

B

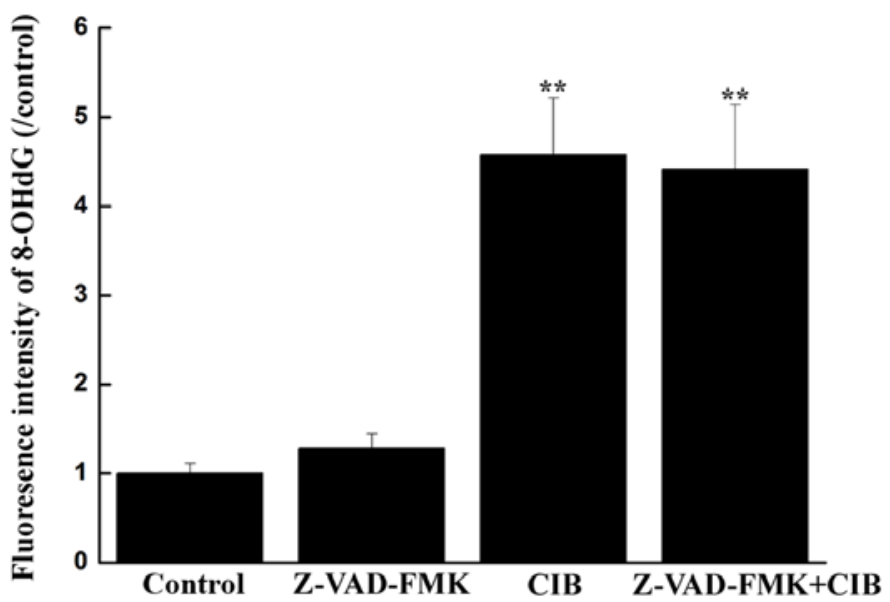

Figure 5. Effect of Z-VAD-FMK and CIB on the oxidative DNA damage. U251 cells were treated with CIB alone, or Z-VAD-FMK alone, or pre-administration with Z-VAD-FMK $(50 \mu \mathrm{M})$ for $1 \mathrm{~h}$ before irradiation. An antibody specific for 8-OHdG lesions was used to visualize DNA oxidative adducts by confocal microscopy. (A) Representative immunofluorescent images of 8-OHdG (red) and DAPI (blue) staining. (B) Relative fluorescence intensity of 8-OHdG is presented as means \pm SD from three independent experiments. ${ }^{* *} \mathrm{P}<0.01$ compared to the control.

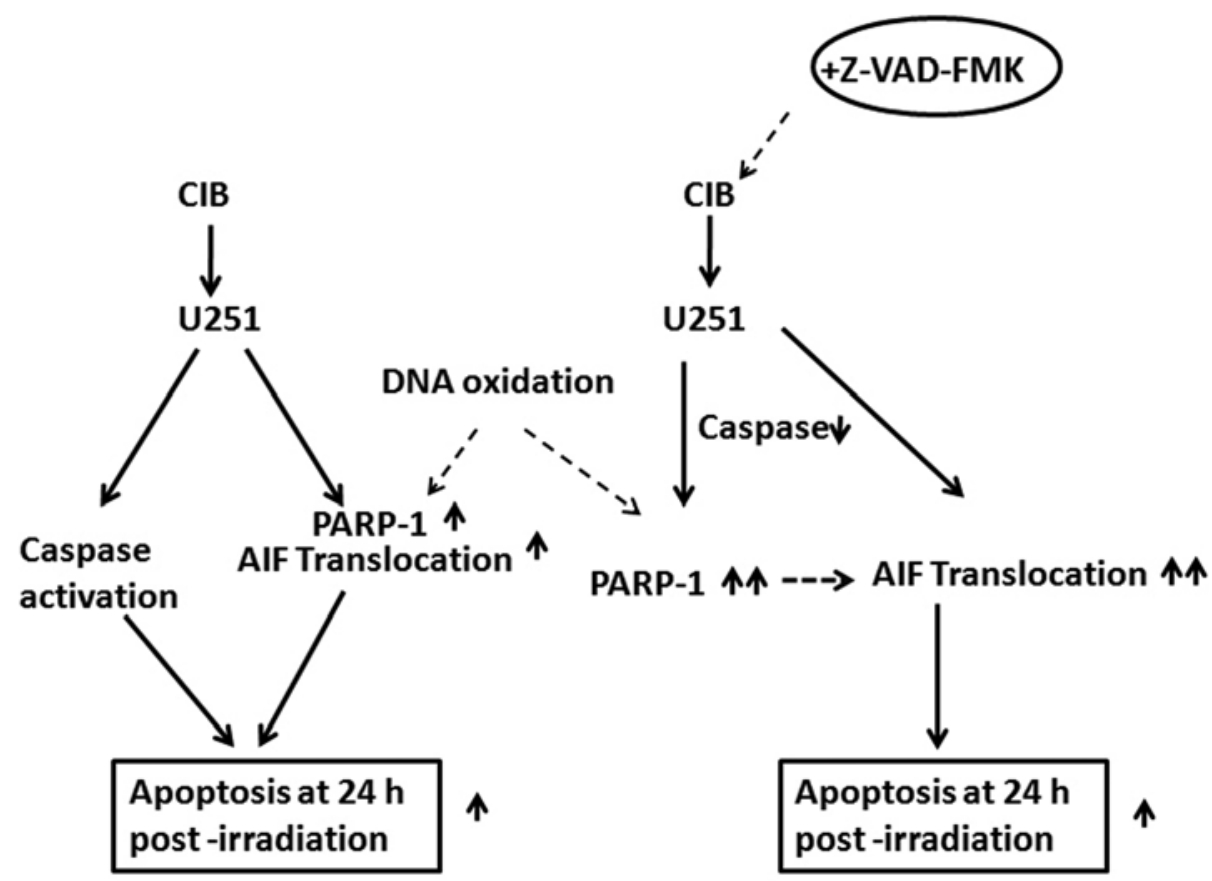

Figure 6. Schematic diagram of the influences of caspase inhibitior Z-VAD-FMK and CIB on the apoptosis pathway in U25 glioma cells. U251 cells were treated with CIB alone, or Z-VAD-FMK alone, or pre-administration with Z-VAD-FMK $(50 \mu \mathrm{M})$ for $1 \mathrm{~h}$ prior to the irradiation. Z-VAD-FMK supplementation suppressed caspase activation and elevated PARP-1 expression. The combination of Z-VAD-FMK with CIB irradiation significantly triggered the AIF nuclear translocation through PARP-1 regulation which was possibly in response to the accumulated oxidative DNA damage. Our findings strongly demonstrated that caspase-independent cell apoptosis provided a prominent compensation in the glioma cell death induced by CIB irradiation.

cell types $(22,23)$. To evaluate whether high-LET CIB irradiation-induced apoptosis is caspase-dependent or not, we immediately applied glioma cells with $50 \mu \mathrm{M}$ of Z-VAD-FMK, a pan-caspase inhibitor before irradiation with $2 \mathrm{~Gy}$ in the present study. The fraction of apoptosis measured at $24 \mathrm{~h}$ following CIB irradiation showed that pre-administration with Z-VAD-FMK did not significantly reduce, in particular, the percentage of Annexin V staining cells (early apoptotic indicator, right lower quadrant of Fig. 1B), suggesting that caspase-independent cell death at an early stage could be triggered effectively by high-LET CIB. Similarly, the data from Ghorai et al also found the existence of caspase-independent 
signaling pathway of apoptosis in cervical cancer HeLa cells caused by CIB irradiation (7).

For further insight into the caspase-independent apoptotic signal evoked by high-LET CIB, we consider that AIF as an important factor involved in the regulation of the glioma cell death. There is strong evidence that AIF translocation occurred in response to neuronal stimuli, including hypoxia, cerebral ischemia and traumatic brain injury $(13,24)$. Our results demonstrate that CIB prominently promoted the mitochondrial release and translocation of AIF to the nucleus visualized using a FlowSight, suggesting that CIB could activate AIF-associated caspase-independent apoptosis, and contribute to the execution of cell death. In particular, the nuclear translocation of AIF was facilitated when caspase activation was suppressed. It is likely that the early activation of caspase caused the inactivation of the process responsible for AIF nuclear translocation (25). Commonly, activation of PARP-1, calpain, cytochrome c, Bax has been reported to affect the mitochondrio-nuclear translocation of AIF $(11,26)$.

However, the molecular mechanism of mitochondrial AIF release to the nucleus induced by high-LET CIB remains obscure. PARP-1 activation is proposed to require translocation of AIF from the mitochondria to the nucleus and that AIF is necessary for PARP-1-dependent cell death (parthanatos) $(18,27)$. In the present study, the levels of PARP-1 mRNA and protein were significantly upregulated by CIB irradiation as seen in the Figs. 3 and 4. Furthermore, an obvious difference of PARP-1 activity raised by CIB irradiation was observed in glioma cells cultured in the presence and absence of Z-VAD-FMK $(\mathrm{P}<0.01)$. Prabhakaran et al also reported that inhibition of caspase cascade with Z-VAD-FMK led to a marked increase of PARP-1 in cyanide-treated cortical cells (28). Moreover, the administration of Z-VAD-FMK enhanced 11'-deoxyverticillin A-elicited PAR formation (29) which played a pivotal role in PARP-1 induced cell death (30). In addition, our data showed a positive correlation between PARP-1 activity and AIF translocation frequency, suggesting that PARP-1 may be strongly activated in glioma cells where it caused the CIB-induced AIF translocation into nucleus, which is consistent with the HeLa cells after exposure with CIB (24). As shown in Fig. 4, nuclear colocalization of AIF and PARP-1 positive signals provided further evidence that PARP-1 initiated AIF-mediated glioma cell death with apoptotic features. Some literature reported that oxidative damage to DNA specifically can trigger caspase-independent apoptosis (31). Our data clearly showed that CIB elevated the amount of 8-OHdG and pre-administration with Z-VAD-FMK did not alter radiation-induced oxidative stress to DNA (Fig. 5), and consequently PARP-1 may be activated to involve in base excision repair process that repairs $8-\mathrm{OHdG}$ lesions (32).

Taken together, our results strongly suggested that AIF translocation from the mitochondria to the nucleus governed by the activated PARP-1 could be particularly effective in glioma cells irradiated with high-LET CIB when the caspase protease cascade is inhibited. These findings demonstrated that at $24 \mathrm{~h}$ after treatment with 2 Gy CIB, caspase-independent glioma cell death provide an outstanding contribution in cell death via PARP-1/AIF pathway, which may be involved in DNA oxidative stress (Fig. 6).

\section{Acknowledgements}

This work was supported by grants from the Key Program of National Natural Science Foundation of China (U1432248), Ministry of Science and Technology National Key R\&D Project (2016YFC0904602) and National Natural Science Foundation of China (nos. 1120521, 11305224 and 11575262).

\section{References}

1. Kao GD, Jiang Z, Fernandes AM, Gupta AK and Maity A: Inhibition of phosphatidylinositol-3-OH kinase/Akt signaling impairs DNA repair in glioblastoma cells following ionizing radiation. J Biol Chem 282: 21206-21212, 2007.

2. Zhang H, Li S, Wang XH, Li Q, Wei SH, Gao LY, Zhao WP, $\mathrm{Hu}$ ZG, Mao RS, Xu HS, et al: Results of carbon ion radiotherapy for skin carcinomas in 45 patients. Br J Dermatol 166: 1100-1106, 2012.

3. Jänicke RU, Engels IH, Dunkern T, Kaina B, Schulze-Osthoff K and Porter AG: Ionizing radiation but not anticancer drugs causes cell cycle arrest and failure to activate the mitochondrial death pathway in MCF-7 breast carcinoma cells. Oncogene 20: 5043-5053, 2001.

4. Park MT, Kim MJ, Kang YH, Choi SY, Lee JH, Choi JA, Kang CM, Cho CK, Kang S, Bae S, et al: Phytosphingosine in combination with ionizing radiation enhances apoptotic cell death in radiation-resistant cancer cells through ROS-dependent and -independent AIF release. Blood 105: 1724-1733, 2005.

5. Di CX, Yang LN, Zhang H, An LZ, Zhang X, Ma XF, Sun C, Wang XH, Yang R, Wu ZH, et al: Effects of carbon-ion beam or $\mathrm{X}$-ray irradiation on anti-apoptosis $\triangle \mathrm{Np} 73$ expression in HeLa cells. Gene 515: 208-213, 2013.

6. Liu B, Zhang H, Zhou G, Xie Y, Hao J, Zhou Q, Duan X and Qiu R: Enhanced cell death by AdCMV-p53 after irradiation of HeLa cells with ${ }^{12} \mathrm{C6}^{+}$ions. Eur J Obstet Gynecol Reprod Biol 138: 226-231, 2008.

7. Ghorai A, Sarma A, Bhattacharyya NP and Ghosh U: Carbon ion beam triggers both caspase-dependent and caspase-independent pathway of apoptosis in HeLa and status of PARP-1 controls intensity of apoptosis. Apoptosis 20: 562-580, 2015.

8. Susin SA, Lorenzo HK, Zamzami N, Marzo I, Snow BE, Brothers GM, Mangion J, Jacotot E, Costantini P, Loeffler M, et al: Molecular characterization of mitochondrial apoptosisinducing factor. Nature 397: 441-446, 1999.

9. Joza N, Susin SA, Daugas E, Stanford WL, Cho SK, Li CY, Sasaki T, Elia AJ, Cheng HY, Ravagnan L, et al: Essential role of the mitochondrial apoptosis-inducing factor in programmed cell death. Nature 410: 549-554, 2001.

10. Candé C, Vahsen N, Garrido C and Kroemer G: Apoptosisinducing factor (AIF): Caspase-independent after all. Cell Death Differ 11: 591-595, 2004.

11. Zhang J, Li XX, Bian HJ, Liu XB, Ji XP and Zhang Y: Inhibition of the activity of Rho-kinase reduces cardiomyocyte apoptosis in heart ischemia/reperfusion via suppressing JNK-mediated AIF translocation. Clin Chim Acta 401: 76-80, 2009.

12. Ferrand-Drake M, Zhu C, Gidö G, Hansen AJ, Karlsson JO, Bahr BA, Zamzami N, Kroemer G, Chan PH, Wieloch T, et al: Cyclosporin A prevents calpain activation despite increased intracellular calcium concentrations, as well as translocation of apoptosis-inducing factor, cytochrome $\mathrm{c}$ and caspase-3 activation in neurons exposed to transient hypoglycemia. J Neurochem 85: 1431-1442, 2003.

13. Zhang X, Chen J, Graham SH, Du L, Kochanek PM, Draviam R, Guo F, Nathaniel PD, Szabó C, Watkins SC, et al: Intranuclear localization of apoptosis-inducing factor (AIF) and large scale DNA fragmentation after traumatic brain injury in rats and in neuronal cultures exposed to peroxynitrite. J Neurochem 82: 181-191, 2002.

14. Hangen E, Blomgren K, Bénit P, Kroemer G and Modjtahedi N: Life with or without AIF. Trends Biochem Sci 35: 278-287, 2010.

15. Oláh G, Szczesny B, Brunyánszki A, López-García IA, Gerö D, Radák Z and Szabo C: Differentiation-associated downregulation of poly(ADP-ribose) polymerase-1 expression in myoblasts serves to increase their resistance to oxidative stress. PLoS One 10: e0134227, 2015. 
16. Kolthur-Seetharam U, Dantzer F, McBurney MW, de Murcia G and Sassone-Corsi P: Control of AIF-mediated cell death by the functional interplay of SIRT1 and PARP-1 in response to DNA damage. Cell Cycle 5: 873-877, 2006.

17. Hong SJ, Dawson TM and Dawson VL: Nuclear and mitochondrial conversations in cell death: PARP-1 and AIF signaling. Trends Pharmacol Sci 25: 259-264, 2004.

18. Yu SW, Wang H, Poitras MF, Coombs C, Bowers WJ, Federoff HJ, PoirierGG, Dawson TM and Dawson VL: Mediation of poly(ADPribose) polymerase-1-dependent cell death by apoptosis-inducing factor. Science 297: 259-263, 2002.

19. Carruthers R and Chalmers AJ: Combination of PARP inhibitors with clinical radiotherapy. Cancer Drug Discov Dev 83: 533-551, 2015.

20. Wieler S, Gagné JP, Vaziri H, Poirier GG and Benchimol S: Poly(ADP-ribose) polymerase-1 is a positive regulator of the p53-mediated G1 arrest response following ionizing radiation. J Biol Chem 278: 18914-18921, 2003.

21. Chen ZT, Zhao W, Qu S, Li L, Lu XD, Su F, Liang ZG, Guo SY and Zhu XD: PARP-1 promotes autophagy via the AMPK/ mTOR pathway in CNE-2 human nasopharyngeal carcinoma cells following ionizing radiation, while inhibition of autophagy contributes to the radiation sensitization of CNE-2 cells. Mol Med Rep 12: 1868-1876, 2015

22. Cummings BS, Kinsey GR, Bolchoz LJ and Schnellmann RG: Identification of caspase-independent apoptosis in epithelial and cancer cells. J Pharmacol Exp Ther 310: 126-134, 2004.

23. Jo GH, Bögler O, Chwae YJ, Yoo H, Lee SH, Park JB, Kim YJ, Kim JH and Gwak HS: Radiation-induced autophagy contributes to cell death and induces apoptosis partly in malignant glioma cells. Cancer Res Treat 47: 221-241, 2015.

24. Ghorai A, Sarma A, Bhattacharyya NP and Ghosh U: Carbon ion beam triggers both caspase-dependent and caspase-independent pathway of apoptosis in HeLa and status of PARP-1 controls intensity of apoptosis. Apoptosis 20: 562-580, 2015.
25. Cregan SP, Fortin A, MacLaurin JG, Callaghan SM, Cecconi F, Yu SW, Dawson TM, Dawson VL, Park DS, Kroemer G, et al: Apoptosis-inducing factor is involved in the regulation of caspase-independent neuronal cell death. J Cell Biol 158: 507-517, 2002.

26. Kondo K, Obitsu S, Ohta S, Matsunami K, Otsuka H and Teshima R: Poly(ADP-ribose) polymerase (PARP)-1-independent apoptosis-inducing factor (AIF) release and cell death are induced by eleostearic acid and blocked by $\alpha$-tocopherol and MEK inhibition. J Biol Chem 285: 13079-13091, 2010.

27. Wang Y, Kim NS, Haince JF, Kang HC, David KK, Andrabi SA, Poirier GG, Dawson VL and Dawson TM: Poly(ADP-ribose) (PAR) binding to apoptosis-inducing factor is critical for PAR polymerase-1-dependent cell death (parthanatos). Sci Signal 4: ra20, 2011.

28. Prabhakaran K, Li L, Borowitz JL and Isom GE: Caspase inhibition switches the mode of cell death induced by cyanide by enhancing reactive oxygen species generation and PARP-1 activation. Toxicol Appl Pharmacol 195: 194-202, 2004.

29. Zhang N, Chen Y, Jiang R, Li E, Chen X, Xi Z, Guo Y, Liu X, Zhou Y, Che Y, et al: PARP and RIP 1 are required for autophagy induced by 11 -deoxyverticillin $\mathrm{A}$, which precedes caspasedependent apoptosis. Autophagy 7: 598-612, 2011.

30. Jin W, Xu W, Chen J, Zhang X, Shi L and Ren C: Remote limb preconditioning protects against ischemia-induced neuronal death through ameliorating neuronal oxidative DNA damage and parthanatos. J Neurol Sci 366: 8-17, 2016.

31. Linsenbardt AJ, Breckenridge JM, Wilken GH and Macarthur H: Dopaminochrome induces caspase-independent apoptosis in the mesencephalic cell line, MN9D. J Neurochem 122: 175-184, 2012.

32. Ding W, Liu W, Cooper KL, Qin XJ, de Souza Bergo PL, Hudson LG and Liu KJ: Inhibition of poly(ADP-ribose) polymerase-1 by arsenite interferes with repair of oxidative DNA damage. J Biol Chem 284: 6809-6817, 2009. 